Bull. Korean Math. Soc. 50 (2013), No. 3, pp. 823-832

http://dx.doi.org/10.4134/BKMS.2013.50.3.823

\title{
A NEW PROJECTION ALGORITHM FOR SOLVING A SYSTEM OF NONLINEAR EQUATIONS WITH CONVEX CONSTRAINTS
}

\author{
LIAN ZHENG
}

\begin{abstract}
We present a new algorithm for solving a system of nonlinear equations with convex constraints which combines proximal point and projection methodologies. Compared with the existing projection methods for solving the problem, we use a different system of linear equations to obtain the proximal point; and moreover, at the step of getting next iterate, our projection way and projection region are also different. Based on the Armijo-type line search procedure, a new hyperplane is introduced. Using the separate property of hyperplane, the new algorithm is proved to be globally convergent under much weaker assumptions than monotone or more generally pseudomonotone. We study the convergence rate of the iterative sequence under very mild error bound conditions.
\end{abstract}

\section{Introduction}

Let $F: \mathbb{R}^{n} \rightarrow \mathbb{R}^{n}$ be a continuous mapping and $C \subset \mathbb{R}^{n}$ be a nonempty closed convex set. Consider the problem of finding $x^{*} \in C$ such that

$$
F\left(x^{*}\right)=0 .
$$

Let $S$ denote the solution set of problem (1). Throughout this paper, we assume that $S$ is nonempty and $F$ has the property that

$$
\left\langle F(y), y-x^{*}\right\rangle \geq 0 \quad \text { for all } y \in C \text { and all } x^{*} \in S,
$$

where $\langle\cdot, \cdot\rangle$ denotes the usual inner product in $\mathbb{R}^{n}$. The property (2) holds if $F$ is monotone or more generally pseudomontone on $C$ in the sense of Karamardian [8].

Nonlinear equations have wide applications in reality. For example, many problems arising from chemical technology, economy and communications can

Received March 8, 2012; Revised September 3, 2012.

2010 Mathematics Subject Classification. 90C25, 90C33.

Key words and phrases. nonlinear equations, projection algorithm, global convergence, convergence rate.

This work was supported by the Educational Science Foundation of Chongqing, Chongqing of China (grant KJ111309). 
be transformed into nonlinear equations (see $[1,2,10,16])$. The popular methods for problem (1) with smooth mapping $F$ may be the Levenberg-Marquardt type method and the trust region method. Under a certain condition, the Levenberg-Marquardt method can be regarded as a special trust region method. Many papers also consider the relations between Levenberg-Marquardt method and trust region method. For more details, please see [3, 4, 5, 13, 19].

Based on this fact that the well-known projection algorithm is a very efficient approach for solving variational inequalities, see $[6,7,17]$. Recently, the literature [15] proposed a projection method for solving problem (1), which possesses a very nice global convergence property without the differentiability or locally Lipschitz continuity assumptions. The numerical performances given in [15] show that the projection method for solving problem (1) is really efficient and has strong stability. More recently, the literatures [9, 14] proposed, respectively, a modified version for the method by changing the projection region in order to accelerate the convergence rate. However, the literatures $[9,15,14]$ require the mapping $F$ is monotone, which seems too stringent a requirement for the purpose of ensuring global convergence property of the projection method.

In this paper, we would introduce a different projection algorithm inspired by He's work for solving variational inequalities in [7]. In the proof of global convergence of our method, the underlying mapping $F$ need only to satisfy the property (2) which is much weaker than monotone or more generally pseudomontone. Moreover, under weaker assumptions than those of $[12,15,14]$, the local rate of convergence of the iterative sequence is established.

The remaining part of this paper is distributed as follows. In the next section, we give some preliminaries and the details of the algorithm. The global convergence analysis of the method is proved in Section 3. The convergence rate of the method will be established in the last section.

\section{Preliminaries and algorithms}

For a nonempty closed convex set $\Omega \subset \mathbb{R}^{n}$ and a vector $x \in \mathbb{R}^{n}$, the projection of $x$ onto $\Omega$ is defined as:

$$
\Pi_{\Omega}(x)=\arg \min \{\|y-x\| \mid y \in \Omega\} .
$$

We have the following properties on the projection operator, see [18].

Lemma 2.1. Let $\Omega \subset \mathbb{R}^{n}$ be a closed convex set. Then it holds that

$$
\left\|x-\Pi_{\Omega}(y)\right\|^{2} \leq\|x-y\|^{2}-\left\|y-\Pi_{\Omega}(y)\right\|^{2}, \forall x \in \Omega, y \in \mathbb{R}^{n} .
$$

Lemma 2.2. Let $C$ be a closed convex set in $\mathbb{R}^{n}, h$ be a real-valued function on $\mathbb{R}^{n}$ and $K$ be the set $\{x \in C: h(x) \leq 0\}$. If $K$ is nonempty and $h$ is Lipschitz continuous on $C$ with modulus $\theta>0$, then

$$
\operatorname{dist}(x, K) \geq \theta^{-1} \max \{h(x), 0\} \text { for all } x \in C,
$$

where $\operatorname{dist}(x, K)$ denotes the distance from $x$ to $K$. 
Proof. See [7, Lemma 2.3].

Algorithm 2.1. Choose $x_{0} \in C, \sigma \in[0,1), \lambda, \beta \in(0,1), a, b \geq 0, a^{2}+b^{2} \neq$ $0, \bar{\mu}>1$. Set $k=0$.

Step 1. Compute $F\left(x_{k}\right)$. If $F\left(x_{k}\right)=0$, stop; Otherwise

Step 2. Choose $\mu_{k} \in[1, \bar{\mu}]$. Compute $\bar{x}_{k} \in \mathbb{R}^{n}$ such that

$$
F\left(x_{k}\right)+\mu_{k}\left(\bar{x}_{k}-x_{k}\right)=r_{k},
$$

where

$$
\left\|r_{k}\right\| \leq \sigma\left\|x_{k}-\bar{x}_{k}\right\| .
$$

Stop if $\bar{x}_{k}=x_{k}$; Otherwise

Step 3. Compute $y_{k}=x_{k}+t_{k}\left(\bar{x}_{k}-x_{k}\right)$, where $t_{k}=\beta^{m_{k}}$ and $m_{k}$ is the smallest nonnegative integer $m$ satisfying

$$
\left\langle F\left(x_{k}+\beta^{m}\left(\bar{x}_{k}-x_{k}\right)\right), x_{k}-\bar{x}_{k}\right\rangle \geq \lambda\left(\mu_{k}-\sigma\right)\left\|x_{k}-\bar{x}_{k}\right\|^{2} .
$$

Step 4. Compute

$$
x_{k+1}=\Pi_{C_{k}}\left(x_{k}\right),
$$

where $C_{k}=\left\{x \in C: h_{k}(x) \leq 0\right\}$ and

$$
h_{k}(x)=\left\langle a F\left(x_{k}\right)+b F\left(y_{k}\right), x-y_{k}\right\rangle+a t_{k}\left\langle F\left(x_{k}\right), \bar{x}_{k}-x_{k}\right\rangle .
$$

Let $k=k+1$ and return to Step 1 .

Remark 2.1. Let $\mu>0$ and $G: \mathbb{R}^{n} \rightarrow \mathbb{R}^{n}$ be a continuous function. When taking

$$
F(x)=r_{\mu}(x)=x-\Pi_{C}(x-\mu G(x)), \bar{x}_{k}=\Pi_{C}\left(x_{k}-\mu G\left(x_{k}\right)\right),
$$

and $\mu_{k} \in[1,1+\sigma]$, our Algorithm 2.1 degrades into the following algorithm.

Algorithm 2.2. Choose $x_{0} \in C, \theta, \beta \in(0,1), a, b \geq 0, a^{2}+b^{2} \neq 0$. Set $k=0$.

Step 1. Compute $r_{\mu}\left(x_{k}\right)$. If $r_{\mu}\left(x_{k}\right)=0$, stop; Otherwise

Step 2. Compute $y_{k}=x_{k}-t_{k} r_{\mu}\left(x_{k}\right)$, where $t_{k}=\beta^{m_{k}}$ and $m_{k}$ is the smallest nonnegative integer $m$ satisfying

$$
\left\langle r_{\mu}\left(x_{k}-\beta^{m} r_{\mu}\left(x_{k}\right)\right), r_{\mu}\left(x_{k}\right)\right\rangle \geq \theta\left\|r_{\mu}\left(x_{k}\right)\right\|^{2} .
$$

Step 3. Compute

$$
x_{k+1}=\Pi_{C_{k}}\left(x_{k}\right),
$$

where $C_{k}=\left\{x \in C: h_{k}(x) \leq 0\right\}$ and

$$
h_{k}(x)=\left\langle a r_{\mu}\left(x_{k}\right)+b r_{\mu}\left(y_{k}\right), x-y_{k}\right\rangle-a t_{k}\left\|r_{\mu}\left(x_{k}\right)\right\|^{2} .
$$

Let $k=k+1$ and return to Step 1 . 
Remark 2.2. A well-known fact is that the solution set of the following variational inequality to find $x^{*} \in C$ such that

$$
\left\langle G\left(x^{*}\right), y-x^{*}\right\rangle \geq 0 \text { for all } y \in C,
$$

coincides with the root of the natural residual function $r_{\mu}($.$) . Therefore, our$ Algorithm 2.2 present a new projection algorithm for problem (9).

Now we analyze the feasibility of Algorithm 2.1. It is obvious that $\bar{x}_{k}$ satisfying conditions (3) and (4) exists. In fact, when we take $\bar{x}_{k}=x_{k}-\frac{1}{\mu_{k}} F\left(x_{k}\right)$, then $\bar{x}_{k}$ satisfies (3) and (4). Next, we need only to show the feasibility of (5).

Lemma 2.3. For all nonnegative integer $k$, there exists a nonnegative integer $m_{k}$ satisfying $(5)$.

Proof. If $\bar{x}_{k}=x_{k}$, then it follows from (4) that $r_{k}=0$, and by (3), we have $F\left(x_{k}\right)=0$. Which means Algorithm 2.1 terminates with $x_{k}$ being a solution of problem (1).

Now, we assume that $\bar{x}_{k} \neq x_{k}$ for all $k$. By the definition of $r_{k}$, we have

$$
\begin{aligned}
\left\langle F\left(x_{k}\right), x_{k}-\bar{x}_{k}\right\rangle & =\mu_{k}\left(x_{k}-\bar{x}_{k}\right)^{T}\left(x_{k}-\bar{x}_{k}\right)+\left(x_{k}-\bar{x}_{k}\right)^{T} \cdot r_{k} \\
& \geq \mu_{k}\left\|x_{k}-\bar{x}_{k}\right\|^{2}-\left\|r_{k}\right\|\left\|x_{k}-\bar{x}_{k}\right\| \\
& \geq\left(\mu_{k}-\sigma\right)\left\|x_{k}-\bar{x}_{k}\right\|^{2} .
\end{aligned}
$$

Now, we suppose that the conclusion of Lemma 2.3 doesn't hold, then there exists a nonnegative integer $k_{0}$ such that (5) is not satisfied for any nonnegative integer $m$, i.e.,

$$
\left\langle F\left(x_{k_{0}}+\beta^{m}\left(\bar{x}_{k_{0}}-x_{k_{0}}\right)\right), x_{k_{0}}-\bar{x}_{k_{0}}\right\rangle<\lambda\left(\mu_{k_{0}}-\sigma\right)\left\|x_{k_{0}}-\bar{x}_{k_{0}}\right\|^{2}, \quad \forall m .
$$

Letting $m \rightarrow \infty$ and by the continuity of $F$, we have

$$
\left\langle F\left(x_{k_{0}}\right), x_{k_{0}}-\bar{x}_{k_{0}}\right\rangle \leq \lambda\left(\mu_{k_{0}}-\sigma\right)\left\|x_{k_{0}}-\bar{x}_{k_{0}}\right\|^{2} .
$$

From (10) and $\bar{x}_{k} \neq x_{k}$, we conclude that $\lambda \geq 1$, which contradicts the fact that $\lambda \in(0,1)$. This completes the proof.

\section{Convergence analysis}

In this section, we first prove two lemmas which are important for the convergence analysis, and then analyze the global convergence of Algorithm 2.1.

Lemma 3.1. If the sequences $\left\{x_{k}\right\}$ and $\left\{y_{k}\right\}$ are generated by Algorithm 2.1, $\left\{x_{k}\right\}$ is bounded and $F$ is continuous, then $\left\{y_{k}\right\}$ is also bounded.

Proof. By (3) and (4), we have

$$
\left\|F\left(x_{k}\right)+\mu_{k}\left(\bar{x}_{k}-x_{k}\right)\right\| \leq \sigma\left\|x_{k}-\bar{x}_{k}\right\| .
$$

Since

It follows that

$$
\mu_{k}\left\|x_{k}-\bar{x}_{k}\right\|-\left\|F\left(x_{k}\right)\right\| \leq\left\|F\left(x_{k}\right)+\mu_{k}\left(\bar{x}_{k}-x_{k}\right)\right\| .
$$

$$
\left(\mu_{k}-\sigma\right)\left\|x_{k}-\bar{x}_{k}\right\| \leq\left\|F\left(x_{k}\right)\right\| .
$$


By the boundedness of $\left\{x_{k}\right\}$, the continuity of $F$ and $\mu_{k} \geq 1>\sigma$, we conclude that $\left\{\bar{x}_{k}\right\}$ is bounded and hence $\left\{y_{k}\right\}$ is bounded.

Lemma 3.2. Let $x^{*}$ be a solution of problem (1), condition (2) hold and the function $h_{k}$ be defined by (6). Then

$$
h_{k}\left(x_{k}\right) \geq t_{k} b \lambda\left(\mu_{k}-\sigma\right)\left\|x_{k}-\bar{x}_{k}\right\|^{2} \text { and } h_{k}\left(x^{*}\right) \leq 0 .
$$

In particular, if $x_{k} \neq \bar{x}_{k}$, then $h_{k}\left(x_{k}\right)>0$.

Proof.

$$
\begin{aligned}
h_{k}\left(x_{k}\right) & =\left\langle a F\left(x_{k}\right)+b F\left(y_{k}\right), x_{k}-y_{k}\right\rangle+a t_{k}\left\langle F\left(x_{k}\right), \bar{x}_{k}-x_{k}\right\rangle \\
& =\left\langle a F\left(x_{k}\right), t_{k}\left(x_{k}-\bar{x}_{k}\right)\right\rangle+b\left\langle F\left(y_{k}\right), x_{k}-y_{k}\right\rangle+a t_{k}\left\langle F\left(x_{k}\right), \bar{x}_{k}-x_{k}\right\rangle \\
(12) \quad & b\left\langle F\left(y_{k}\right), x_{k}-y_{k}\right\rangle \\
& =t_{k} b\left\langle F\left(y_{k}\right), x_{k}-\bar{x}_{k}\right\rangle \\
(13) \quad & \geq t_{k} b \lambda\left(\mu_{k}-\sigma\right)\left\|x_{k}-\bar{x}_{k}\right\|^{2},
\end{aligned}
$$

where the inequality follows from (5).

$$
\begin{aligned}
h_{k}\left(x^{*}\right) & =\left\langle a F\left(x_{k}\right)+b F\left(y_{k}\right), x^{*}-y_{k}\right\rangle+a t_{k}\left\langle F\left(x_{k}\right), \bar{x}_{k}-x_{k}\right\rangle \\
& =a\left\langle F\left(x_{k}\right), x^{*}-y_{k}\right\rangle+b\left\langle F\left(y_{k}\right), x^{*}-y_{k}\right\rangle+a t_{k}\left\langle F\left(x_{k}\right), \bar{x}_{k}-x_{k}\right\rangle \\
& \leq a\left\langle F\left(x_{k}\right), x^{*}-x_{k}-t_{k}\left(\bar{x}_{k}-x_{k}\right)\right\rangle+a t_{k}\left\langle F\left(x_{k}\right), \bar{x}_{k}-x_{k}\right\rangle \\
& =a\left\langle F\left(x_{k}\right), x^{*}-x_{k}\right\rangle \\
& \leq 0
\end{aligned}
$$

where the two inequalities follow from condition (2).

If $x_{k} \neq \bar{x}_{k}$, then $h_{k}\left(x_{k}\right)>0$ because $\sigma<1 \leq \mu_{k}$. The proof is completed.

Remark 3.1. Lemma 3.2 means that the hyperplane

$$
H_{k}:=\left\{x \in \mathbb{R}^{n} \mid\left\langle a F\left(x_{k}\right)+b F\left(y_{k}\right), x-y_{k}\right\rangle+a t_{k}\left\langle F\left(x_{k}\right), \bar{x}_{k}-x_{k}\right\rangle=0\right\}
$$

strictly separates the current iterate from the solutions of problem (1).

From the proof of Lemma 3.2, we know that $-\left(a F\left(x_{k}\right)+b F\left(y_{k}\right)\right)$ is a descent direction of the function $\frac{1}{2}\left\|x-x^{*}\right\|^{2}$ at the point $x_{k}$ if $x_{k} \neq \bar{x}_{k}$, where $x^{*} \in S$. In fact,

$$
\begin{aligned}
& \left\langle a F\left(x_{k}\right)+b F\left(y_{k}\right), x_{k}-x^{*}\right\rangle \\
= & a\left\langle F\left(x_{k}\right), x_{k}-x^{*}\right\rangle+b\left\langle F\left(y_{k}\right), x_{k}-x^{*}\right\rangle \\
= & a\left\langle F\left(x_{k}\right), x_{k}-x^{*}\right\rangle+b\left\langle F\left(y_{k}\right), x_{k}-y_{k}\right\rangle+b\left\langle F\left(y_{k}\right), y_{k}-x^{*}\right\rangle \\
\geq & b\left\langle F\left(y_{k}\right), x_{k}-y_{k}\right\rangle \\
\geq & t_{k} b \lambda\left(\mu_{k}-\sigma\right)\left\|x_{k}-\bar{x}_{k}\right\|^{2} \\
> & 0 .
\end{aligned}
$$

Where the first inequality follows from condition (2), the second one follows from (12) and (13), and the last one follows $x_{k} \neq \bar{x}_{k}$. 
We next prove our main convergence result. Certainly, if Algorithm 2.1 terminates at Step $k$, then $x_{k}$ is a solution of problem (1). So, in the following analysis, we assume that Algorithm 2.1 always generates an infinite sequence.

Theorem 3.1. If $F$ is continuous on $C$ and condition (2) holds, then the sequence $\left\{x_{k}\right\} \subset \mathbb{R}^{n}$ generated by Algorithm 2.1 globally converges to a solution of problem (1).

Proof. Let $x^{*}$ be a solution of problem (1). Since $x_{k+1}=\Pi_{C_{k}}\left(x_{k}\right)$, it follows from Lemma 2.1 that

$$
\begin{aligned}
\left\|x_{k+1}-x^{*}\right\|^{2} & \leq\left\|x_{k}-x^{*}\right\|^{2}-\left\|x_{k}-x_{k+1}\right\|^{2} \\
& =\left\|x_{k}-x^{*}\right\|^{2}-\operatorname{dist}^{2}\left(x_{k}, C_{k}\right),
\end{aligned}
$$

which shows that the sequence $\left\{\left\|x_{k+1}-x^{*}\right\|\right\}$ is nonincreasing, and hence is a convergent sequence. Therefore, $\left\{x_{k}\right\}$ is bounded and

$$
\lim _{k \rightarrow \infty} \operatorname{dist}\left(x_{k}, C_{k}\right)=0 .
$$

From Lemma 3.1 and the continuity of $F$, we have $\left\{F\left(x_{k}\right)\right\}$ and $\left\{F\left(y_{k}\right)\right\}$ are bounded, that is, for some $M>0$,

$$
\left\|a F\left(x_{k}\right)+b F\left(y_{k}\right)\right\| \leq M, \forall k .
$$

So,

$$
\begin{aligned}
\left|h_{k}(x)-h_{k}(y)\right| & =\left|\left\langle a F\left(x_{k}\right)+b F\left(y_{k}\right), x-y\right\rangle\right| \\
& \leq\left\|a F\left(x_{k}\right)+b F\left(y_{k}\right)\right\|\|x-y\| \\
& \leq M\|x-y\|, \quad \forall x, y \in \mathbb{R}^{n},
\end{aligned}
$$

which shows that $h_{k}$ is Lipschitz continuous on $C$ with modulus $M$. Applying Lemma 2.2 and Lemma 3.2 and noting that $x_{k} \notin C_{k}$. we obtain that

$$
\begin{aligned}
\operatorname{dist}\left(x_{k}, C_{k}\right) & \geq M^{-1} h_{k}\left(x_{k}\right) \\
& \geq M^{-1} b \lambda\left(\mu_{k}-\sigma\right) t_{k}\left\|x_{k}-\bar{x}_{k}\right\|^{2}, \forall k .
\end{aligned}
$$

By (15) and (16), it holds that

$$
\lim _{k \rightarrow \infty} t_{k}\left\|x_{k}-\bar{x}_{k}\right\|^{2}=0
$$

If $\lim \sup _{k \rightarrow \infty} t_{k}>0$, then we must have

$$
\liminf _{k \rightarrow \infty}\left\|x_{k}-\bar{x}_{k}\right\|^{2}=0 \text {. }
$$

Hence, by (4), we have

$$
\liminf _{k \rightarrow \infty} r_{k}=0 .
$$

Since $\left\{x_{k}\right\}$ is bounded, the sequence has at least one cluster point. Let $\hat{x}$ be a cluster point of $\left\{x_{k}\right\}$ and $\left\{x_{k_{i}}\right\}$ be the corresponding convergent subsequence. By the definition of $r_{k}$, one has

$$
r_{k_{i}}=F\left(x_{k_{i}}\right)+\mu_{k_{i}}\left(\bar{x}_{k_{i}}-x_{k_{i}}\right),
$$


letting $i \rightarrow \infty$, we have $F(\hat{x})=0$ because $F$ is continuous and $\left\{\mu_{k}\right\}$ is bounded. This implies that $\hat{x}$ is a solution of problem (1). Replacing $x^{*}$ by $\hat{x}$ in (14), we obtain that the sequence $\left\{\left\|x_{k}-\hat{x}\right\|\right\}$ is nonincreasing and hence converges. Moreover, the subsequence $\left\{\left\|x_{k_{i}}-\hat{x}\right\|\right\}$ converges to zero. which shows that the whole sequence $\left\{\left\|x_{k}-\hat{x}\right\|\right\}$ converges to zero, and hence $\lim _{k \rightarrow \infty} x_{k}=\hat{x}$.

Suppose now that $\lim _{k \rightarrow \infty} t_{k}=0$. Let $\bar{x}$ be any accumulation point of $\left\{x_{k}\right\}$, there exists some subsequence $\left\{x_{k_{j}}\right\}$ converging to $\bar{x}$. By the choice of $t_{k},(5)$ implies that

$$
\begin{aligned}
\lambda\left(\mu_{k_{j}}-\sigma\right)\left\|x_{k_{j}}-\bar{x}_{k_{j}}\right\|^{2} & >\left\langle F\left(x_{k_{j}}+\beta^{m_{k_{j}}-1}\left(\bar{x}_{k_{j}}-x_{k_{j}}\right)\right), x_{k_{j}}-\bar{x}_{k_{j}}\right\rangle \\
& =\left\langle F\left(x_{k_{j}}+\frac{1}{\beta} t_{k_{j}}\left(\bar{x}_{k_{j}}-x_{k_{j}}\right)\right), x_{k_{j}}-\bar{x}_{k_{j}}\right\rangle .
\end{aligned}
$$

From (10) and (17), we have

$$
\begin{aligned}
& \left(\mu_{k_{j}}-\sigma\right)\left\|x_{k_{j}}-\bar{x}_{k_{j}}\right\|^{2}+\left\langle F\left(x_{k_{j}}+\frac{1}{\beta} t_{k_{j}}\left(\bar{x}_{k_{j}}-x_{k_{j}}\right)\right)-F\left(x_{k_{j}}\right), x_{k_{j}}-\bar{x}_{k_{j}}\right\rangle \\
< & \lambda\left(\mu_{k_{j}}-\sigma\right)\left\|x_{k_{j}}-\bar{x}_{k_{j}}\right\|^{2},
\end{aligned}
$$

letting $j \rightarrow \infty$ and using the continuity of $F$, we have

$$
\left.\left.\lim _{j \rightarrow \infty} \| x_{k_{j}}-\bar{x}_{k_{j}}\right)\left\|^{2} \leq \lambda \lim _{j \rightarrow \infty}\right\| x_{k_{j}}-\bar{x}_{k_{j}}\right) \|^{2} .
$$

Since $\lambda \in(0,1)$, we conclude that

$$
\left.\lim _{j \rightarrow \infty} \| x_{k_{j}}-\bar{x}_{k_{j}}\right) \|=0 .
$$

Using the similar argument to that of the first case, we conclude that every accumulate point of $\left\{x_{k}\right\}$ is a solution of problem (1).

\section{Convergence rate}

By Theorem 3.1, we know that the generated sequence $\left\{x^{k}\right\}$ converges to a solution of problem (1). Throughout this section, we always assume that $x^{k} \rightarrow x^{*}$, where $x^{*} \in S$, as $k \rightarrow \infty$. To establish the result on the convergence rate of the iterative sequence generated by Algorithm 2.1, we need the following conditions (18) and (19).

For $x^{*} \in S$, there exist three poisitive constants $\delta, c$ and $L$ such that

$$
c \operatorname{dist}(x, S) \leq\|F(x)\|, \forall x \in N\left(x^{*}, \delta\right),
$$

$$
\|F(x)-F(y)\| \leq L\|x-y\|, \forall x, y \in N\left(x^{*}, \delta\right),
$$

where $\operatorname{dist}(x, S)$ denotes the distance from $x$ to the solution set $S$, and

$$
N\left(x^{*}, \delta\right)=\left\{x \in R^{n} \mid\left\|x-x^{*}\right\| \leq \delta\right\} .
$$

If $F$ is differentiable and $\nabla F(\cdot)$ is locally Lipschitz continuous with modulus $\theta$, then there exists a positive constant $L_{1}$ such that

$$
\|F(y)-F(x)-\nabla F(x)(y-x)\| \leq L_{1}\|y-x\|^{2}, \forall x, y \in N\left(x^{*}, \delta\right) .
$$


In fact, by the mean value theorem of vector valued function, we have

$$
\begin{aligned}
& \|F(y)-F(x)-\nabla F(x)(y-x)\| \\
= & \left\|\int_{0}^{1} \nabla F(\tau y+(1-\tau) x)(y-x) d \tau-\int_{0}^{1} \nabla F(x)(y-x) d \tau\right\| \\
\leq & \int_{0}^{1}\|\nabla F(\tau y+(1-\tau) x)-\nabla F(x)\|\|y-x\| d \tau \\
\leq & \theta\|y-x\|^{2} \int_{0}^{1} \tau d \tau \\
= & L_{1}\|y-x\|^{2}
\end{aligned}
$$

where $L_{1}=\theta / 2$. In $(20)$, replacing $\nabla F(x)$ with $G_{k}$ which is a positive semidefinite matrix, we obtain

$$
\left\|F(y)-F(x)-G_{k}(y-x)\right\| \leq L_{1}\|y-x\|^{2}, \forall x, y \in N\left(x^{*}, \delta\right) .
$$

Under the condition (20) or (21), it is easily shown that the condition (19) holds. In other words, (19) is weaker than (20) or (21). In 1998, the literature [12] showed that their proposed method converged superlinearly when the underlying function $F$ is differentiable with $\nabla F\left(x^{*}\right)$ nonsingular and $\nabla F$ is locally Lipschitz continuous. It is known that the local error bound condition given in (18) is weaker than the nonsingular. Recently, under the conditions (18) and (21), the literature [15] obtained the local linear rate of convergence of the proposed method.

Next, we analyze the convergence rate of the iterative sequence under the conditions (18) and (19).

Theorem 4.1. In addition to the assumptions in the Theorem 3.1, if conditions (18)-(19) hold, then there is a constant $\omega>0$ such that for sufficiently large $k$,

$$
\operatorname{dist}\left(x_{k}, S\right) \leq \frac{1}{\sqrt{\omega k+\operatorname{dist}^{-2}\left(x_{0}, S\right)}}
$$

Proof. By (10), we have

$$
\left(\mu_{k}-\sigma\right)\left\|x_{k}-\bar{x}_{k}\right\|^{2} \leq\left\langle F\left(x_{k}\right), x_{k}-\bar{x}_{k}\right\rangle,
$$

letting $k \rightarrow \infty$, from the continuity of $F$, Theorem 3.1 and $\mu_{k} \geq 1>\sigma$, it follows that

$$
\lim _{k \rightarrow \infty}\left\|x_{k}-\bar{x}_{k}\right\|=0
$$

Therefore, $x_{k}+\beta^{-1} t_{k}\left(\bar{x}_{k}-x_{k}\right) \in N\left(x^{*}, \delta\right)$ for $k$ sufficiently large because the sequence $\left\{x_{k}\right\}$ converges to $x^{*}$.

By the construction of $t_{k}$, we have

$$
\left\langle F\left(x_{k}+\beta^{-1} t_{k}\left(\bar{x}_{k}-x_{k}\right)\right), x_{k}-\bar{x}_{k}\right\rangle<\lambda\left(\mu_{k}-\sigma\right)\left\|x_{k}-\bar{x}_{k}\right\|^{2} .
$$

From (22) and (23), we obtain

(24) $\left\langle F\left(x_{k}\right)-F\left(x_{k}+\beta^{-1} t_{k}\left(\bar{x}_{k}-x_{k}\right)\right), x_{k}-\bar{x}_{k}\right\rangle>(1-\lambda)\left(\mu_{k}-\sigma\right)\left\|x_{k}-\bar{x}_{k}\right\|^{2}$. 
Using the Cauchy-Schwarz inequality and the condition (19), for $k$ large enough it holds that

$$
\left\langle F\left(x_{k}\right)-F\left(x_{k}+\beta^{-1} t_{k}\left(\bar{x}_{k}-x_{k}\right)\right), x_{k}-\bar{x}_{k}\right\rangle \leq L \beta^{-1} t_{k}\left\|x_{k}-\bar{x}_{k}\right\|^{2} .
$$

By (24) and (25) and $\mu_{k} \geq 1$, for $k$ sufficiently large we obtain

$$
t_{k}>\beta(1-\lambda)(1-\sigma) / L \text {. }
$$

By $(3)$ and $1 \leq \mu_{k} \leq \bar{\mu}$, we have

$$
\begin{aligned}
\left\|F\left(x_{k}\right)\right\| & \leq\left\|r_{k}\right\|+\mu_{k}\left\|x_{k}-\bar{x}_{k}\right\| \\
& \leq\left(\sigma+\mu_{k}\right)\left\|x_{k}-\bar{x}_{k}\right\| \\
& \leq(\sigma+\bar{\mu})\left\|x_{k}-\bar{x}_{k}\right\| .
\end{aligned}
$$

Therefore, by the condition (18), we have

$$
\left\|x_{k}-\bar{x}_{k}\right\| \geq \frac{c}{\sigma+\bar{\mu}} \operatorname{dist}\left(x_{k}, S\right) .
$$

Let $z_{k} \in S$ be the closest solution to $x_{k}$. That is, $\left\|x_{k}-z_{k}\right\|=\operatorname{dist}\left(x_{k}, S\right)$. By (14), (16), (26) and (27), we obtain that for sufficiently large $k$,

$$
\begin{aligned}
& \operatorname{dist}^{2}\left(x_{k+1}, S\right) \\
\leq & \left\|x_{k+1}-z_{k}\right\|^{2} \\
\leq & \left\|x_{k}-z_{k}\right\|^{2}-M^{-2} b^{2} \lambda^{2}\left(\mu_{k}-\sigma\right)^{2} t_{k}^{2}\left\|x_{k}-\bar{x}_{k}\right\|^{4} \\
< & \left\|x_{k}-z_{k}\right\|^{2}-M^{-2} L^{-2} b^{2} \beta^{2} \lambda^{2}(1-\lambda)^{2}(1-\sigma)^{4}\left\|x_{k}-\bar{x}_{k}\right\|^{4} \\
\leq & \operatorname{dist}^{2}\left(x_{k}, S\right)-M^{-2} L^{-2}(\sigma+\bar{\mu})^{-4} b^{2} c^{4} \beta^{2} \lambda^{2}(1-\lambda)^{2}(1-\sigma)^{4} \operatorname{dist}^{4}\left(x_{k}, S\right) .
\end{aligned}
$$

Let

$$
\omega=M^{-2} L^{-2}(\sigma+\bar{\mu})^{-4} c^{4} b^{2} \beta^{2} \lambda^{2}(1-\lambda)^{2}(1-\sigma)^{4} .
$$

Applying Lemma 6 in [11, Chapter 2], we obtain

$$
\begin{aligned}
\operatorname{dist}\left(x_{k}, S\right) & \leq \operatorname{dist}\left(x_{0}, S\right) / \sqrt{\omega k \operatorname{dist}^{2}\left(x_{0}, S\right)+1} \\
& =1 / \sqrt{\omega k+\operatorname{dist}^{-2}\left(x_{0}, S\right)} .
\end{aligned}
$$

The completes the proof.

\section{References}

[1] S. P. Dirkse and M. C. Ferris, MCPLIB: A collection of nonlinear mixed complementarity problems, Optim. Methods Software 5 (1995), 319-345.

[2] M. E. EL-Hawary, Optimal Power Flow: Solution Techniques, Requirement and Challenges, IEEE Service Center, Piscataway, NJ, 1996.

[3] J. Y. Fan, Convergence rate of the trust region method for nonlinear equations under local error bound condition, Comput. Optim. Appl. 34 (2006), no. 2, 215-227.

[4] J. Y. Fan and J. Y. Pan, An improved trust region algorithm for nonlinear equations, Comput. Optim. Appl. 48 (2011), no. 1, 59-70.

[5] J. Y. Fan and Y. X. Yuan, On the quadratic convergence of the Levenberg-Marquardt method without nonsingularity assumption, Computing 74 (2005), no. 1, 23-39. 
[6] D. R. Han, A hybrid entropic proximal decomposition method with self-adaptive strategy for solving variational inequality problems, Comput. Math. Appl. 55 (2008), no. 1, 101115 .

[7] Y. R. He, A new double projection algorithm for variational inequalities, J. Comput. Appl. Math. 185 (2006), no. 1, 166-173.

[8] S. Karamardian, Complementarity problems over cones with monotone and pseudomonotone maps, J. Optim. Theory Appl. 18 (1976), no. 4, 445-454.

[9] F. M. Ma and C. W. Wang, Modified projection method for solving a system of monotone equations with convex constraints, J. Appl. Math. Comput. 34 (2010), no. 1-2, 47-56.

[10] K. Meintjes and A. P. Morgan, A methodology for solving chemical equilibrium system, Appl. Math. Comput. 22 (1987), no. 4, 333-361.

[11] B. T. Polyak, Introduction to Optimization, Optimization Software Inc., Publications Division, New York, 1987(Translated from Russian, with a foreword by Dimitri P. Bertsekas).

[12] M. V. Solodov and B. F. Svaiter, A globally convergent inexact Newton method for systems of monotone equations, In: Fukushima M., Qi L. (eds) Reformulation: piecewise smooth, semismooth and smoothing methods. Kluwer, Holanda (1998), 355-369.

[13] X. J. Tong and L. Qi, On the convergence of a trust-region method for solving constrained nonlinear equations with degenerate solution, J. Optim. Theory Appl. 123 (2004), no. $1,187-211$.

[14] C. W. Wang and Y. J. Wang, A superlinearly convergent projection method for constrained systems of nonlinear equations, J. Global Optim. 44 (2009), no. 2, 283-296.

[15] C. W. Wang, Y. J. Wang, and C. L. Xu, A projection method for a system of nonlinear monotone equations with convex constraints, Math. Methods Oper. Res. 66 (2007), no. $1,33-46$.

[16] A. J. Wood and B. F. Wollenberg, Power Generations, Operations, and Control, Wiley, New York, 1996

[17] N. Xiu and J. Zhang, Some recent advances in projection-type methods for variational inequalities, J. Comput. Appl. Math. 152 (2003), no. 1-2, 559-585.

[18] E. H. Zarantonello, Projections on Convex Sets in Hilbert Spaces and Spectral Theory, Academic Press, New York, 1971.

[19] J. L. Zhang and Y. Wang, A new trust region method for nonlinear equations, Math. Methods Oper. Res. 58 (2003), no. 2, 283-298.

Department of Mathematics and Computer Science

YANGTZE NORMAL UnIVERSITY

Fuling, Chongqing 408100, P. R. China

E-mail address: zhenglian66@yahoo.com.cn 